\title{
Axo-Axonic Structures in the Medial Prefrontal Cortex of the Rat: Reduction by Prenatal Exposure to Cocaine
}

\author{
Bret A. Morrow, John D. Elsworth, and Robert H. Roth \\ Neuropsychopharmacology Research Unit, Departments of Pharmacology and Psychiatry, Yale University School of Medicine, New Haven, \\ Connecticut 06520-8066
}

\begin{abstract}
The cognitive deficits associated with prenatal exposure to cocaine have been hypothesized to be the results of changes in the anatomy and function of the frontal cortex. In this study, pregnant dams were treated with cocaine $(3 \mathrm{mg} / \mathrm{kg}$ i.v. twice a day) and the resulting adolescent (postnatal day, $\sim 45$ ) male offspring were killed for immunocytochemical determination of the total linear measure, number, location, and lengths of inhibitory GABA transporter-1 immunoreactive axo-axonic structures commonly called "candles" or "cartridges" in the medial prefrontal cortex. These inhibitory structures are the axon terminals of GABAergic cells that impinge on the initial axon segments of excitatory pyramidal neurons. We report that prenatal cocaine exposure decreased the number of these inhibitory candles. The greatest reduction of candles was observed in the ventral prelimbic cortex. Additionally, there was a subtle difference in the pattern of distribution of candles, namely the depth of the initial candle in the ventral portions of the prefrontal cortex was greater in rats exposed to prenatal cocaine. However, there was no overt change in the number of cells that were immunoreactive for the calcium-binding protein parvalbumin, an indicator of a subset of GABAergic interneurons that includes axo-axonic chandelier cells. We conclude that exposure to cocaine in utero disrupts the development of the axo-axonic cells in the prefrontal cortex and this disruption could contribute to the cognitive deficits reported with prenatal cocaine exposure.
\end{abstract}

Key words: development; GABA transporter-1; GAT-1; dopamine; cartridge; candle

\section{Introduction}

Prenatal exposure to cocaine disrupts cognitive function in both humans (Heffelfinger et al., 1997; Mayes et al., 1998; Leech et al., 1999) and in associated animal models (Heyser et al., 1995; Garavan et al., 2000; Morrow et al., 2002b). The operational mechanism of this disruption is not clear, although it may involve the neuronal systems of the prefrontal cortex (PFC). Several lines of data support this hypothesis. First, some cognitive disruption, such as that seen in humans including schizophrenic patients, is thought to involve alteration in the function of the prefrontal cortex. Second, in animal models, cocaine exposure has resulted in several changes to the structure and function of the prefrontal cortex: elevated stress-induced dopamine metabolism (Elsworth et al., 2001; Morrow et al., 2001), enhanced numbers of GABAcontaining interneurons (Wang et al., 1995), and altered dendritic fields of a subgroup of the GABAergic interneurons (Wang et al., 1996; Stanwood et al., 2001). Finally, we have recently reported that rats prenatally exposed to cocaine demonstrate a hyper-reactivity of the intrinsic neurons in the medial prefrontal cortex, as indicated by the exaggerated expression of the immediate-early gene $c$-fos (Morrow et al., 2002a).

These data seem to indicate a disruption in the excitatory/ inhibitory balance of this cortical region in prenatal cocaineexposed rats. To further investigate this possibility, we chose to examine the inhibitory axo-axonic structures commonly called

\footnotetext{
Received April 1, 2002; revised April 3, 2003; accepted April 11, 2003.

This work was supported by National Institute of Drug Abuse Grant DA-11288. We thank Dottie Cameron for her excellent technical work and creative use of sewing utensils in stereology.

Correspondence should be addressed to Dr. Bret Morrow, Department of Pharmacology, Yale University School of Medicine, 333 Cedar Street, New Haven, C 06520-8066. E-mail: bret.morrow@yale.edu.

Copyright $\odot 2003$ Society for Neuroscience $\quad$ 0270-6474/03/235227-08\$15.00/0
}

“candles" or "cartridges" (DeFelipe et al., 1985; Peters et al., 1990; Lewis, 2000). A candle is the terminal of an axon of a GABAergic interneuron that impinges on the initial axon segment of a pyramidal neuron. The primary source of these axo-axonic structures is the chandelier cell, but other interneurons appear to be the source of some of the candles (Peters and Harriman, 1992). From this anatomical location, these neurons potentially can exert an inhibitory influence that is disproportional to their number. Lewis (2000) recently reported a diminished number of axoaxonic structures in the brains of schizophrenic subjects, indicating that candles may have a role in the cognitive dysfunction associated with this disorder (Lewis, 2000; Volk et al., 2001).

The primary objective of this study was to determine whether prenatal exposure to cocaine diminished the total linear measure of these candles in the medial prefrontal cortex. In addition to the primary objective, several secondary endpoints were examined, including the number, average length, and location of these axoaxonic candles. Additionally, the number of neurons immunostained for the calcium-binding protein parvalbumin (PV) was estimated. PV can be used to identify a subset of GABAergic neurons that includes chandelier cells, the axons of which make up the candles, and basket cells (Gabbott and Bacon, 1996).

\section{Materials and Methods}

Animals and tissue collection. Pregnant dams were given saline or cocaine ( $3 \mathrm{mg} / \mathrm{kg}$ i.v. twice a day) from embryonic day (E) 10 to E20, as described previously (Morrow et al., 2001, 2002a). The offspring from each treated dam were fostered to a nontreated nursing dam at birth and then weaned at P21 and allowed to mature to a late adolescent/young adult stage. Prenatal saline and cocaine-exposed rats were killed on P42, P45, or P48. No differences in GAT-1-IR were noted between these ages. This preadult age was selected to better mimic the juvenile ages when cognitive 
effects have occurred in humans exposed to cocaine in utero (DelaneyBlack et al., 1998; Dow-Edwards et al., 1999). Brain tissues were collected from seven cocaine-exposed male offspring and five saline-exposed male offspring, each from a different dam.

Rats were give pentobarbital ( $65 \mathrm{mg} / \mathrm{kg}$ i.p.; Sigma, St. Louis, MO) to induce deep anesthesia and vascularly perfused with $50 \mathrm{ml}$ of saline with heparin $(1 \mathrm{U} / \mathrm{ml})$ followed by $250 \mathrm{ml}$ of $4 \%$ formaldehyde in phosphate buffer ( $\mathrm{PB} ; 0.1 \mathrm{M}, \mathrm{pH}$ 7.4). The brains were stored overnight in $4 \%$ formaldehyde in $\mathrm{PB}$ and then cut on a freezing stage microtome into 40 $\mu \mathrm{m}$ coronal sections and serially separated into five sets of tissues so that each set had tissue sections $200 \mu \mathrm{m}(5 \times 40 \mu \mathrm{m}$ tissue section $)$ apart. This provided a random uniform sampling technique with regard to the rostral-caudal plane or $z$-axis (Howard and Reed, 1998). A set of tissue sections from $\sim 2.7-3.5 \mathrm{~mm}$ anterior to bregma was collected for analysis. This area of the frontal cortex was selected because of the strong activation of c-fos observed in prenatal cocaine-exposed rats (Morrow et al., 2002a). In an attempt to get as many complete candles as possible, the tissue was cut in coronal sections. Candles are nearly exclusively located perpendicular to the cortical surface. All tissue from control and cocaineexposed rats were immunostained at the same time and all investigators handling the tissue were blinded to the animal's treatment until the data were collected.

Immunostaining. Endogenous peroxidase-like activity was quenched in the tissue sections with $0.01 \%$ hydrogen peroxide in $\mathrm{PB}$ for $10 \mathrm{~min}$ and rinsed repeatedly in PB. A polyclonal antibody (AB1570, Lot 18070063; Chemicon, Temecula, CA) directed against the c-terminus (aa 588-599) of the GABA transporter-1 (GAT-1) was used to identify the axo-axonic candles. Whereas antibodies directed against GABA and PV can also be used to identify these structures, antibodies against GAT-1 can provide a clear identification of the structures with less collateral staining than that seen with antibodies directed against GABA and PV (Gabbott and Bacon, 1996; Gabbott et al., 1997; Lewis, 2000). Free-floating tissue sections were incubated in rabbit anti-GAT-1 (1:500 in PB with $0.5 \%$ Triton X-100 and $10 \%$ normal goat serum) overnight at room temperature. Note that a substantial variation in the concentration and immunostaining conditions was observed between lots of antibody. Sections were then washed in $\mathrm{PB}$, incubated at room temperature for $2 \mathrm{hr}$ in a biotinylated goat anti-rabbit secondary antibody (1:222 in PB; Vector Laboratories, Burlingame, $\mathrm{CA}$ ), rinsed with $\mathrm{PB}$, and finally incubated in an avidin-biotin complex reagent (Vector Laboratories) at one-half the suggested concentration of the manufacture for $90 \mathrm{~min}$ at room temperature. The sections were then washed and the tissue-bound peroxidase was visualized using a nickel-intensified diaminobenzidine reaction $(0.04 \%$ diaminobenzidine, $2.5 \%$ nickel sulfate, and $0.005 \%$ hydrogen peroxide) for $4.75 \mathrm{~min}$. Tissue sections were mounted on gelatin-coated slides and stained using $0.1 \%$ cresyl violet (a Nissl stain) to allow visualization of brain landmarks. This protocol provided immunostaining through the entire depth of the $40 \mu \mathrm{m}$ sections. Black and white photomicrographs in Figure 1 demonstrate only the near black GAT-1-IR immunostaining without the lighter purple cresyl violet counterstain for ease of identifying the axoaxonic candles. Each batch of primary or secondary antibody used was tested for nonspecific staining by omission of the appropriate antibody.

Adjacent tissue sections were immunostained to identify the calciumbinding protein PV. A subpopulation of GABAergic interneurons including the chandelier cells, the axon terminals of which make up the majority of the candles, contain PV. Additionally, this subpopulation also includes large and small basket cells. Selective staining of this and other calcium-binding proteins has proven useful in identifying distinct subpopulations of GABAergic interneurons (Gabbott and Bacon, 1996; Gabbott et al., 1997). The procedure was similar to that used for GAT1-IR but used the following antibodies: the primary was directed against PV (P3171; 1:10,000 in PB with 5\% normal horse serum; Sigma) and the secondary was biotinylated horse anti-mouse (1:222 with 5\% normal horse serum; Vector Laboratories). Figure 2 shows black and white photomicrographs of the PV-IR immunostaining without cresyl violet counterstain for clarity.

Sampling technique and quantification. All GAT-1-IR tissues were quantified under oil immersion microscopy (Olympus BH-2 with camera lucida, DPlanApo $40 \times 1.00$ oil; Olympus Optical, Tokyo, Japan).
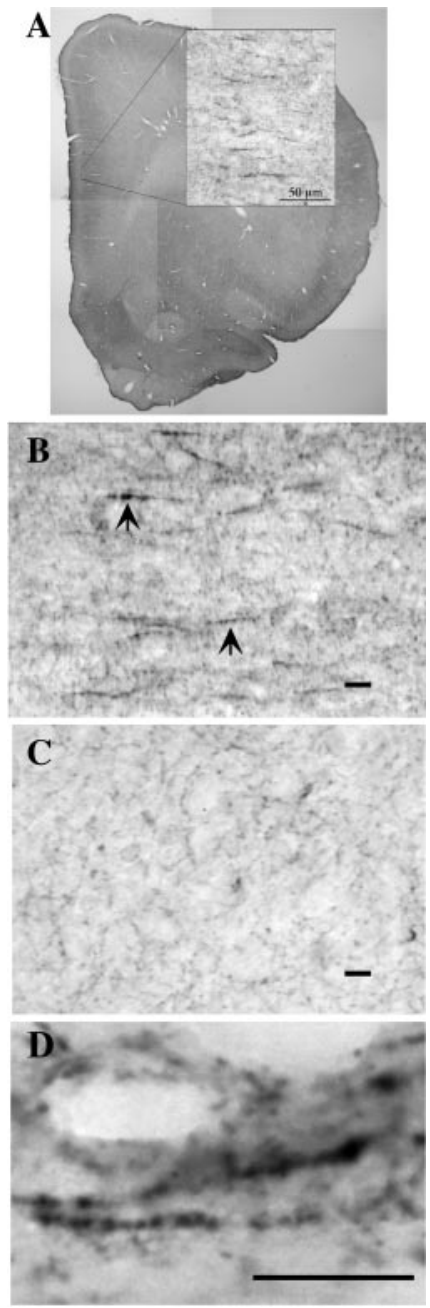

Figure 1. GAT-1 in the medial prefrontal cortex of control adolescent rats. Prepared brain slices $(40 \mu \mathrm{m})$ were immunostained for GAT-1 and counterstained for Nissl. For clarity, the tissues in $B$ and ( photomicrographs were not counterstained. $A$, GAT-1-IR axo-axonic structures are located in the shallow aspects of a band of GAT-1-IR in the medial prefrontal cortex. The inset shows the band of GAT-1-IR at higher magnification. $B$, The GAT-1-IR axo-axonic structures (arrows) display a pattern similar to pearls on a string. C, GAT-1-IR also identified a more difuse punctate pattern within the cortex but not white matter. $D$, Higher magnified image of GAT-1-IR structures. Scale bar, $10 \mu \mathrm{m}$.

Several important observations were made during the preliminary study to optimize the staining and quantification of the GAT-1-IR (data not shown). First, GAT-1-IR demonstrated a diffuse-punctated stain as well as the more distinct candle staining of the axo-axonic structures that has been described previously as resembling pearls on a string (Peters et al., 1990; Lewis, 2000). Second, the axo-axonic GAT-1-IR candle staining was strongest in the medial prefrontal cortex, namely the anterior cingulate $(\mathrm{aCg})$, prelimbic $(\mathrm{PL})$, and infralimbic (IL) cortices with fewer or, in some areas, no candles observed in the dorsal and lateral aspects including the M1 and M2 motor and primary somoatosensory cortices. The more ventral aspects including the orbital and agranular insular cortices demonstrated some GAT-1-IR candles. In contrast, the diffusepunctated GAT-1-IR was present throughout the gray matter of the frontal cortex but displayed a denser wide band in the shallower aspects of the cortex (see Fig. 1). The distribution of the GAT-1-IR candles was nearly exclusively limited to a narrow band $\sim 200 \mu \mathrm{m}$ wide, located in the deep portion of layer II and the shallow aspects of layer III. This band of candles was located in the shallowest aspects of the wide band of diffuse GAT-1-IR (see Fig. 1). Third, GAT-1-IR candles were obscured if a sufficiently deep Nissl stain was used to allow precise identification of the cortical layers. For this reason, a light Nissl stain was used in these exper- 


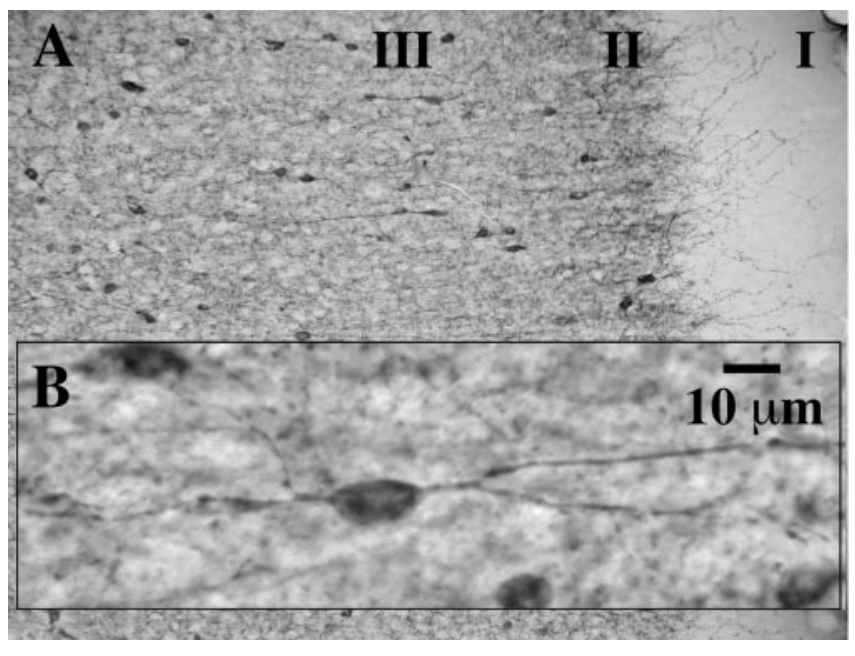

Figure 2. PV-containing neurons in the medial prefrontal cortex. Brain slices adjacent to those stained for GAT-1-IR were immunostained for PV and counterstained for Nissl. The photomicrographs shown here do not have the Nissl counterstain for clarity. A, PV-IR in the shallow regions of the medial prefrontal cortex showing cell bodies and PV-IR fibers. The roman numeral identifies the approximate start of the cortical layer. B, A photomicrograph of a bipolar PV-IR cell in the medial prefrontal cortex. Scale bar, $10 \mu \mathrm{m}$.

iments, and the location of GAT-1-IR candles was determined by the depth from the cortical surface rather than exact cortical layer.

The other observations from the preliminary studies help determine how to quantify the GAT-1-IR candles. The right or left tissue sections were alternatively sampled using a random uniform technique starting above the medial prefrontal cortex and moving $500 \mu \mathrm{m}$ in the $y$-axis, dorsal to ventral, to each sample site. At each sample site, camera lucida drawings (two-dimensional) were made of a $60 \mu \mathrm{m}$ wide sector running perpendicular from the cortical surface to a point well below the end of the band of GAT-1-IR candles, $575 \mu \mathrm{m}$ below the cortical surface. Figure 3 shows examples of camera lucida drawings from the ventral PL (vPL)-IL region of two cocaine-exposed rats and two saline-exposed rats.

We determined all measurements reported here from the camera lucida drawings. The primary objective of this study was accomplished by measuring the total length of GAT-1-IR in each $60 \mu \mathrm{m}$ wide section and averaging all of the samples $[\mathrm{aCg}$, dorsal PL (dPL), vPL, and IL] within each of the five coronal tissue slices (data presented in Figs. 4 and 5). The number of GAT-1-IR candles was determined in a similar manner, but by counting. A secondary, more detailed analysis was performed on two tissue sections ( $\sim 2.9$ and $3.1 \mathrm{~mm}$ anterior to bregma) to determine the length, location within the dorsal to ventral subregions of the medial PFC (mPFC), and depth from the cortical surface of the GAT-1-IR candles (data presented in Figs. 7 and 8, and Table 1). To determine the length of the GAT-1-IR candles, we used a random uniform sampling technique for length (Howard and Reed, 1998) on the $\sim 2.9$ and $3.1 \mathrm{~mm}$ anterior to bregma sections. A dressmaker's pattern-tracing wheel was used to trace the camera lucida drawing leaving "ticks" at a uniform interval. A pattern-tracing tool is basically a small wheel with teeth on the outer edge that can be used to easily trace objects that are not straight. The tool was placed on the paper before the candle started, thus providing a random starting point to avoid potential measurement bias (Howard and Reed, 1998), and traced over the top of the candle for its entire length. We counted the number of ticks on the camera lucida drawing of the candle to estimate the length of each candle. If a candle had four ticks on its camera lucida drawing, it was estimated to be between four and five ticks in length. Candles $<3.5 \mu \mathrm{m}$ were not counted to avoid potential inclusion of pericellular and other noncandle GAT-1-IR clusters.

The methods used in this paper provided a thorough quantitative examination of axo-axonic structures immunostained for GAT-1 in the medial frontal cortex. However, any analysis has certain compromises. The use of a random uniform sampling technique is generally accepted as a less biased method and was used in both the $z$-and $y$-axis. Because the
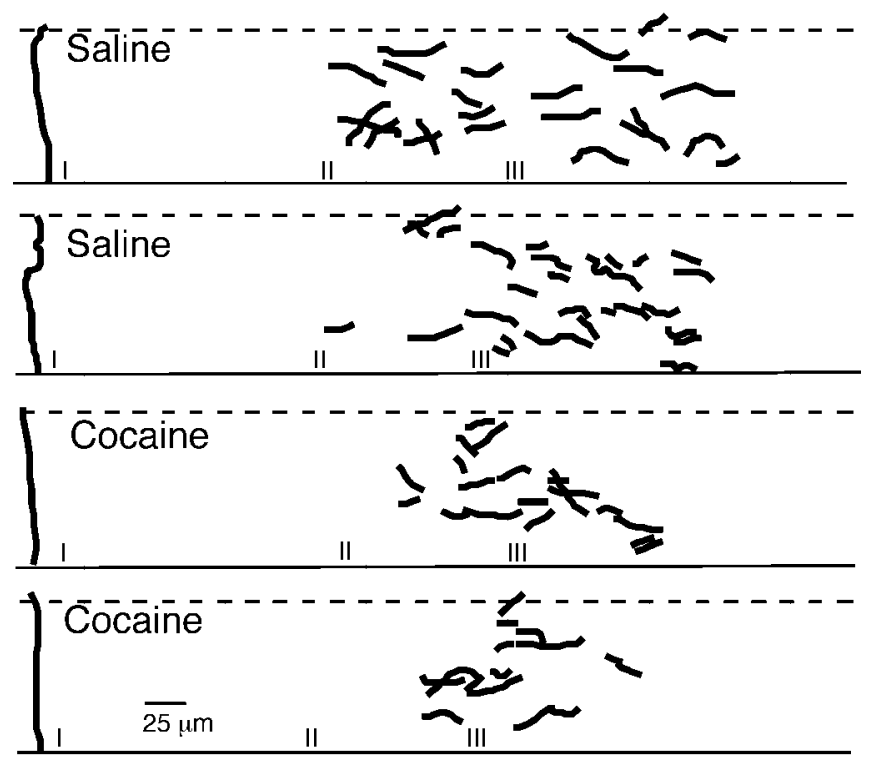

Figure 3. Camera lucida drawings of GAT-1-IR candles in the prelimbic region of the medial prefrontal cortex in four rats prenatally exposed to saline or cocaine. The Roman numeral identifies the beginning of the cortical layer. GAT-1-IR candles crossing the exclusion line (solid) were not measured, whereas those crossing the inclusion line (dashed) were counted.

Table 1. Prenatal exposure to cocaine disrupted the depth (in millimeters) from cortical surface of the initial GAT-1-IR axo-axonic structure

\begin{tabular}{lllll}
\hline Prenatal Tx & $\mathrm{aCg}$ & $\mathrm{dPL}$ & $\mathrm{vPL}$ & $\mathrm{IL}$ \\
\hline Saline & $187 \pm 26$ & $182 \pm 22$ & $131 \pm 20$ & $124 \pm 27$ \\
Cocaine & $190 \pm 8$ & $197 \pm 6$ & $200 \pm 10^{*}$ & $183 \pm 10^{*}$ \\
\hline
\end{tabular}

${ }^{*} p<0.05$ versus saline control value in the same region.

GAT-1-IR candles displayed a distinct distribution primarily in a $175 \mu \mathrm{m}$ wide band in cortical layers II and III, the decision was made to examine the entire section rather than use uniform sampling in the $x$-axis. However, this decision should not add any bias to the analysis. The total linear GAT-1-IR was selected as the primary measure to observe potential cocaine-related changes in either the length or number of GAT-1-IR candles. GAT-1-IR candles within each $40 \mu \mathrm{m}$ tissue section were analyzed using a two-dimensional method rather than a more timeconsuming three-dimensional method. Although this method could potentially result in a bias to overestimate the number of GAT-1-IR candles because of counting partial structures at the cut surfaces at the top and bottom of the section, this would not alter the primary measure (the total linear GAT-1-IR). Additionally, GAT-1-IR axo-axonic structures are principally located perpendicular to the cortical surface and parallel to the cut surfaces and are therefore far less likely to cross the cut surfaces compared with randomly distributed structures. Finally, the selection of any method of sample preparation and analysis can potentially introduce some bias from the absolute true value. However, this bias exists equally in the prenatal saline and cocaine samples and not between them. We believe that the methods selected for this study provide an accurate and precise quantitative analysis of the GAT-1-IR candles in the medial frontal cortex in a timely manner.

The number of PV-IR cells was estimated using a two-dimensional camera lucida technique similar to that used with the GAT-1-IR candles but with some changes attributable to the larger size of the PV-IR cells. The sample width was $343 \mu \mathrm{m}$, and the distance between sampling locations was $1 \mathrm{~mm}$. All five tissue sections were sampled to estimate the number of PV-IR cells. To compare directly with the estimated number of GAT-1-IR candles, the estimated number of PV-IR cells was expressed per $60 \mu \mathrm{m}$ wide sample section.

Statistics. All data are presented as mean $\pm \operatorname{SEM}(n=7$ rats prenatally exposed to cocaine; $n=5$ rats prenatally exposed to saline). Data were 
analyzed using repeated measures ANOVA $(1 \times 1)$ with Newman-Kuels range testing for the between factor, prenatal treatment, and means contrasts for within factors and any significant interactions between the main factors. The dependent factor was different for each analysis: the estimated total linear GAT-1-IR, the estimated number of GAT-1-IR candles, the distribution rostral to caudal, and the estimated number of PV-IR cells. For the secondary analysis, the between factor was always prenatal treatment, and the within factors were length, depth, and dorsal-ventral distribution.

\section{Results}

The axo-axonic projections of chandelier cells have been described previously in the cortex of the rat (Peters et al., 1982; Peters and Harriman, 1990), nonhuman primate (DeFelipe et al., 1985; Ong et al., 1998; Hof et al., 1999), and human (Woo et al., 1998; Pierri et al., 1999). In approaching the primary and secondary objectives of this study, it was necessary to quantitatively describe GAT-1-IR candles in the mPFC of normal (prenatal saline exposed) rats. Our results agree with previous qualitative descriptions of these structures. GAT-1-IR staining resulted in two distinct staining patterns (Fig. 1). The first was a diffuse punctate pattern located primarily in cortical layers II-VI but not in the white matter. GAT-1-IR presented as a darker band in layers II and III, visible to the naked eye (see Fig. $1 A$ ). The second pattern of immunostaining was the more distinct candle staining of the axo-axonic structures. These structures were primarily oriented perpendicular to the cortical surface in the medial and ventral frontal cortex. Fewer or no candles were located in the lateral and dorsal aspects of the frontal cortex. The vast majority of the candles were located within the shallower aspects of the darker staining band of GAT-1-IR in cortical layers II and III. The axo-axonic structures have been described previously as pearls on a string because of the easily identifiable immunoreactive axon fiber and boutons (Peters et al., 1982; DeFelipe et al., 1985; Peters and Harriman, 1990).

\section{Prenatal cocaine reduced the overall linearGAT-1-IR axo-axonic structures}

Prenatal exposure to cocaine resulted in a lower total linear GAT1 -IR at P45 (Fig. 4) $\left(F_{(1,10)}=5.43 ; p=0.04\right)$. All regions sampled were averaged for each rostral-caudal section. The rostral-caudal distribution of GAT-1-IR varied in the MPFC, demonstrating the greatest level at $\sim 3.1 \mathrm{~mm}$ anterior to bregma. There was no significant interaction between prenatal treatment and the rostral-caudal distribution; however, the greatest difference between cocaine- and saline-exposed rats was at the same point, $\sim 3.1 \mathrm{~mm}$ anterior to bregma. The total number of GAT-1-IR candles was counted across the entire MPFC region analyzed, and there was a trend for a reduction associated with the prenatal cocaine treatment (Fig. 5) $\left(F_{(1,10)}=3.98 ; p=0.07\right)$.

\section{Prenatal cocaine did not alter the number of PV-IR cells}

The number of PV-IR cells was also estimated in adjacent tissue slices. Figure 6 shows PV-IR cells and dendritic fibers in the mPFC. There was no effect of prenatal cocaine exposure on the number of PV-IR cells in adjacent sections of the mPFC (Fig. 6) $\left(F_{(1,10)}=0.15 ; p=0.70\right)$. No additional analysis of PV-IR cells was performed.

Prenatal cocaine did not alter the length of GAT-1-IR candles The secondary analysis focused on two sections $(\sim 2.9$ and 3.1 $\mathrm{mm}$ anterior to bregma) that had the greatest amount of GAT1 -IR and the greatest difference between prenatal saline and co-

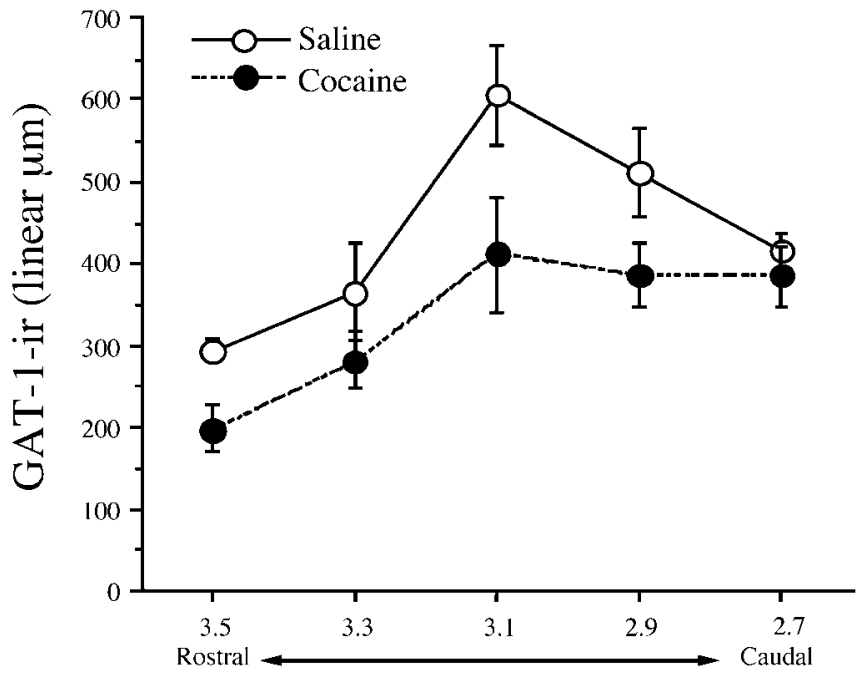

Approximate location in $\mathrm{mm}$ anterior to Bregma

Figure 4. Rats exposed to cocaine in utero have significantly less linear GAT-1-IR per $60 \mu \mathrm{m}$ wide sector in the $\mathrm{MPFC}$. Adolescent ( $\sim$ P45) male offspring from dams that were given saline or cocaine (3 mg/kg i.v. twice daily; E10 -E20) supplied brain tissues that were immunostained GAT-1 and counterstained for Nissl. From a random starting point, $\sim 3.5 \mathrm{~mm}$ anterior to bregma, five sections were taken at $200 \mu \mathrm{m}$ intervals to $\sim 2.7 \mathrm{~mm}$ anterior to bregma. Within each section, the $\mathrm{mPFC}$ was sampled every $500 \mu \mathrm{m}$ starting at a randomly selected point above the MPFC, working dorsal to ventral. At each sample site, lineal GAT-1-IR was measured in a 60 $\mu \mathrm{m}$ wide sector running perpendicular to the cortical surface. All dorsal-ventral sample sites within a single tissue section were averaged. Data were analyzed using $1 \times 1$ repeated measures ANOVA (between factor, prenatal treatment; within factor, location of sample relative to bregma).

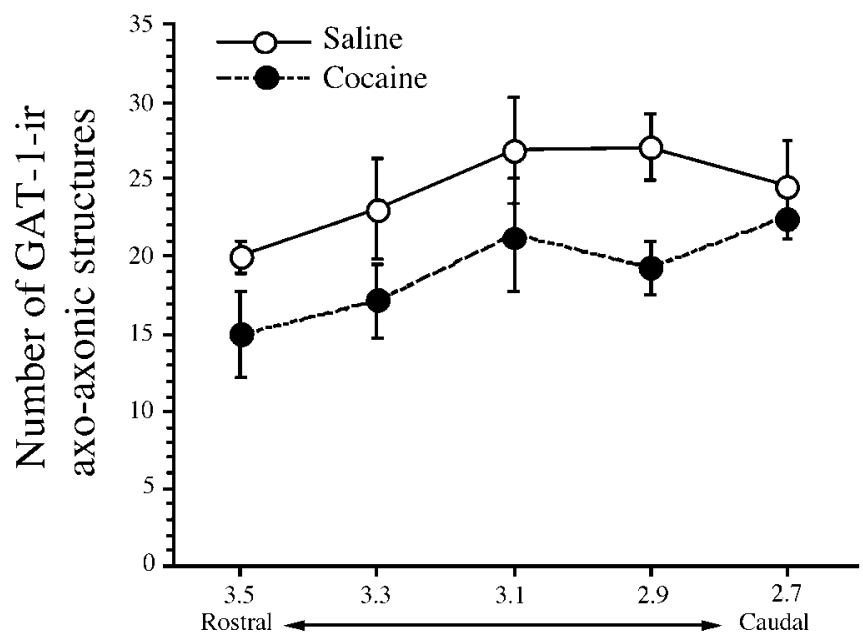

Approximate location in $\mathrm{mm}$ anterior to Bregma

Figure 5. The number of GAT-1-IR axo-axonic structures (candles) was greatest in the middle, rostral to caudal, of the mPFC ( $\sim 2.9-3.1 \mathrm{~mm}$ anterior to bregma). The number of GAT-1-IR candles per $60 \mu \mathrm{m}$ wide sector in each rostral-caudal tissue section was averaged from dorsal-ventral samples taken every $500 \mu \mathrm{m}$. In this gross analysis, there was a trend for an overall decrease in the number of GAT-1-IR candles in the prenatal cocaine-treated rats ( $p=0.07$; $1 \times 1$ repeated measures ANOVA).

caine. The objectives were to examine the distribution of GAT1 -IR candles by length, depth from cortical surface, and dorsalventral location. The length of GAT-1-IR candles in control animals displayed an apparent Gaussian-type distribution with the greatest abundance of GAT-1-IR candles between 7 and 14 $\mu \mathrm{m}$ in length (Fig. 7). Only $\sim 25 \%$ of the candles in control rats were longer than $21 \mu \mathrm{m}$. The overall number of GAT-1-IR can- 


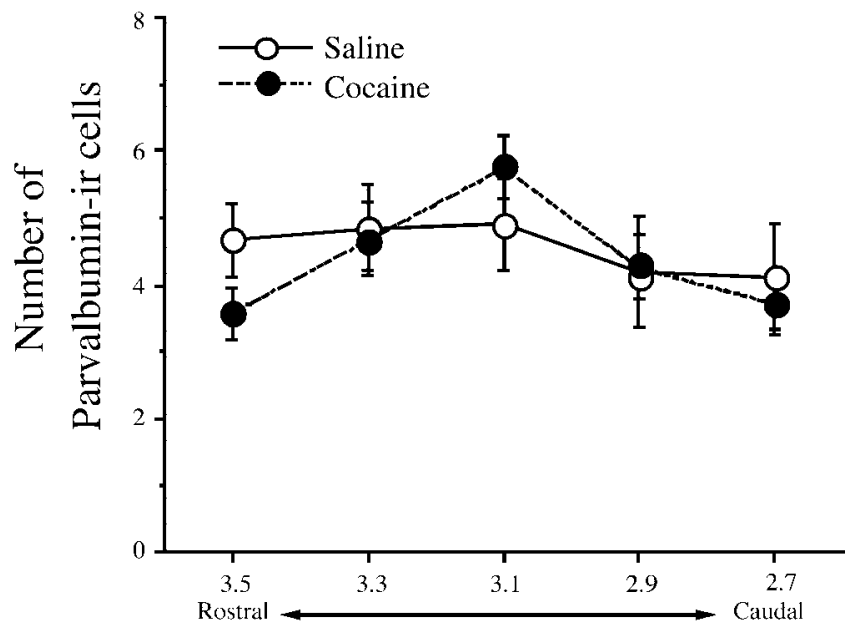

Approximate location in $\mathrm{mm}$ anterior to Bregma

Figure 6. The number of PV-IR cells in the medial prefrontal cortex was not altered by prenatal cocaine exposure. The medial prefrontal cortex of adolelescent $(\sim P 45)$ male rats were immunostained for PV, counterstained for Nissl, and counted for PV-IR cells using a random uniform sampling technique.

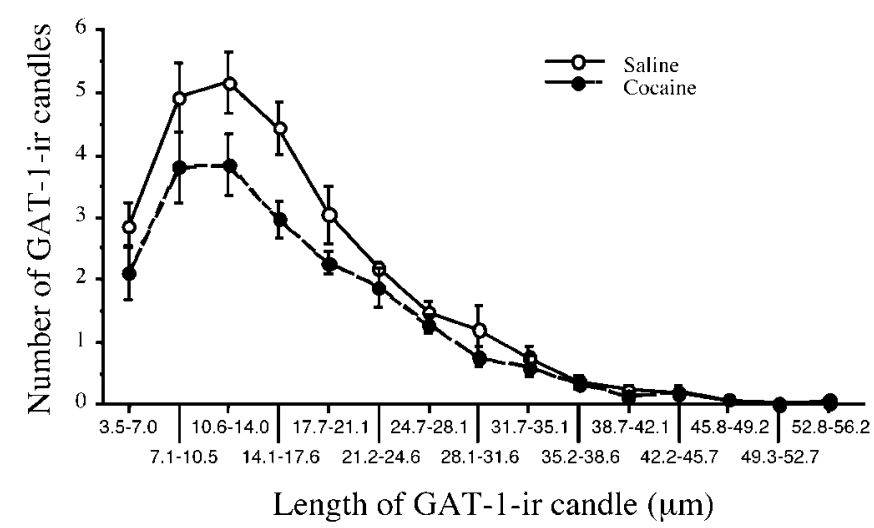

Figure 7. The number, but not lengths, of GAT-1-IR axo-axonic structures was altered by prenatal exposure to cocaine. Two sections with the most GAT-1-IR axo-axonic staining, 3.1 and $2.9 \mathrm{~mm}$ anterior to bregma, were examined for the length of these candle structures using a random uniform sampling technique for length in each animal. In each tissue section, the total numbers of candles in all dorsal-ventral subregions were added together and expressed in the graph by length within a $3.5 \mu \mathrm{m}$ range. Candles $<3.5 \mu \mathrm{m}$ were not counted to avoid potential inclusion of pericellular and other smaller GAT-1-IR clusters. Data were analyzed using repeated measures ANOVA ( $1 \times 1$; between factor, prenatal treatment; within factor, length), demonstrating an overall reduction in the total number of GAT-1-IR candles in the cocaine-exposed offspring but no difference in distribution by length.

dles were reduced in prenatal cocaine-exposed rats $\left(F_{(1,10)}=\right.$ 5.02; $p=0.049$ ), but the median length and the distribution by length was unchanged.

Prenatal cocaine selectively reduced the number of GAT-1-IR candles in vPL cortex

The secondary analysis of control animals indicated that GAT1 -IR candles were nearly exclusively located in layers II and III, with $>90 \%$ located within a band from 176 to $350 \mu \mathrm{m}$ below and running roughly parallel to the cortical surface. In the saline control rats, the depth of the initial candle was reduced in the ventral regions, in which the cortex is thinner. The average depth of the first GAT-1-IR candle in the saline controls varied from 190 to $125 \mu \mathrm{m}$, dorsal to ventral, whereas in the prenatal cocaine-
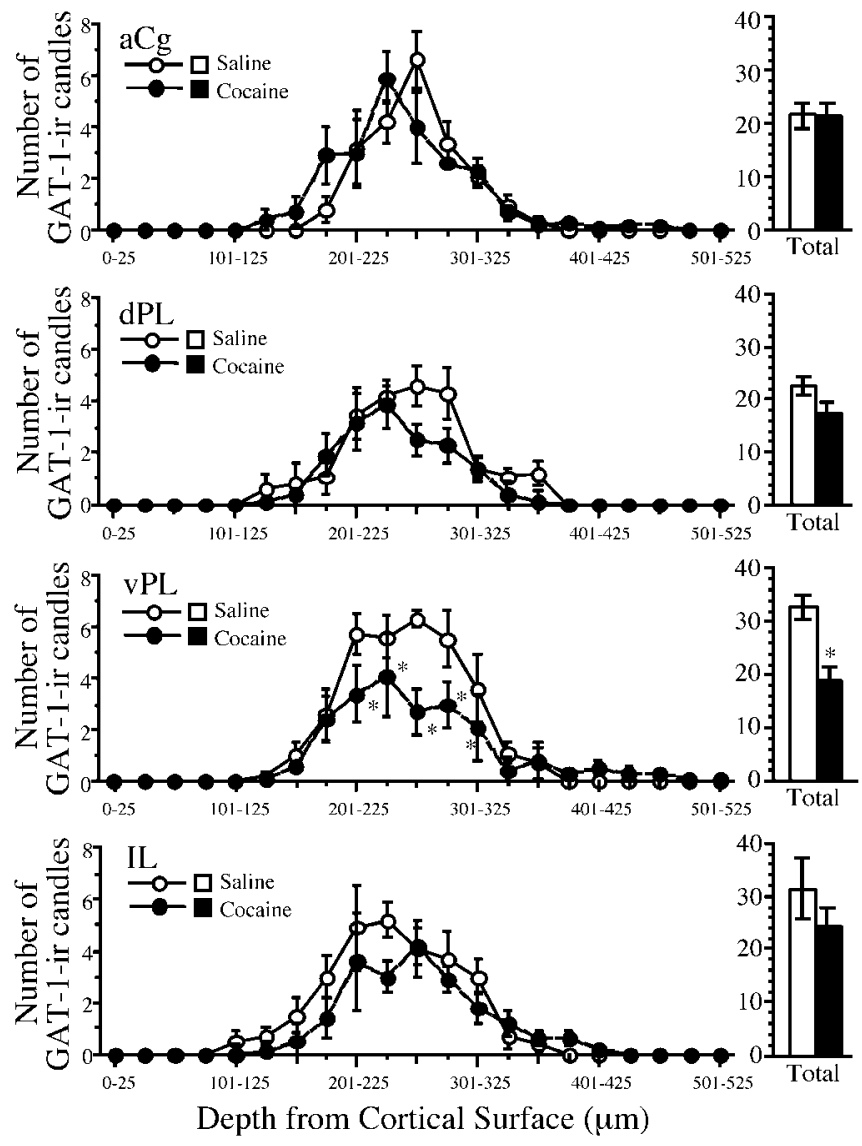

Figure 8. GAT-1-IR axo-axonic structures were reduced the most in the vPL but without an apparent change in the distribution by depth from cortical surface in the two sections with the most GAT-1-IR axo-axonic staining, 3.1 and $2.9 \mathrm{~mm}$ anterior to bregma. GAT-1-IR candles nearly exclusively occupy a band $\sim 175 \mathrm{~mm}$ wide in layers II and III of the medial prefrontal cortex, namely the aCg, dorsal and ventral PL, and IL cortices. Looking dorsal to ventral, the depth of the initial axo-axonic structures was reduced in the saline-exposed controls but not in the cocaine-exposed rats (see Table 1). Data were analyzed using repeated measures ANOVA $(1 \times 1$; between factor, prenatal treatment; within factor, depth)

exposed rat, the depth of the first candle remained at $\sim 190 \mu \mathrm{m}$ below the cortical surface in all mPFC regions examined (Table 1). This alteration in pattern resulted in significant differences in the depth of the first candle in the vPL and IL cortex of prenatal cocaine-exposed rats (Table 1$)\left(F_{(3,30)}=3.56 ; p=0.026\right)$. This effect was also indicated by an analysis of the rate of change in depth of the first candle versus region, dorsal to ventral (saline, slope $=46 \mu \mathrm{m} / \mathrm{mm}, r=0.69$; cocaine, slope $=6 \mu \mathrm{m} / \mathrm{mm}, r=$ $0.17)$.

Finally, the secondary analysis allowed us to compare the saline and cocaine in utero-exposed samples for the location of GAT-1-IR candles in both the $x$ - and $y$-axis, dorsal-ventral and medial-lateral, respectively. Prenatal cocaine exposure was associated with a selective reduction in the number of GAT-1-IR candles in the vPL cortex by $\sim 50 \%$ but not in the aCg or the IL (Fig. 8) (vPL, $F_{(1,10)}=18.14, p=0.002$; aCg, $p=0.25$; IL, $p=$ $0.59)$. There was a trend for a reduction in the number of GAT1 -IR candles in the dPL, but this failed to reach significance $\left(F_{(1,10)}=4.696 ; p=0.055\right)$. No apparent change in the distribution by depth from cortical surface other than that noted above was observed in any region within the $\mathrm{mPFC}$ of prenatal cocaineexposed rats. 


\section{Discussion}

Prenatal cocaine had a number of effects on the axo-axonic GAT1 -IR structures in the medial prefrontal cortex of rats. The total lineal GAT-1-IR was reduced in prenatal cocaine-exposed rats. The reason for the decrease in total lineal GAT-1-IR was a decline in the number of GAT-1-IR axo-axonic structures and not a change in the length of the structures, as determined by either a change in the median length or the distribution by length. The greatest prenatal cocaine-associated reduction was in the ventral PL cortex at $\sim 3 \mathrm{~mm}$ anterior to bregma, the middle of the medial prefrontal cortex, rostral to caudal. Alternatively, the apparent loss of GAT-1-IR candles could have been a result from the loss of the immunoreactive target, the GABA transporter-1, but not the actual axo-axonic structure. In this study, there is no way to determine whether the immunoreactive staining or the actual structure is lost; however, the loss of the GABA transporter-1 could, in itself, lead to diminished function of the candles by altering the reuptake of GABA. There was no apparent reduction in the overall number of PV-IR cells in the $\mathrm{mPFC}$ in the prenatal cocaine-treated rats, leading us to tentatively conclude that the number of GAT-1-IR axo-axonic structures per chandelier cell is also reduced. One caveat is that PV-IR identifies basket cells as well as chandelier cells. A second caveat is that a small subgroup of axo-axonic structures may originate from interneurons other than chandelier cells (Peters and Harriman, 1992). A second, less likely possibility is that a loss of PV-IR chandelier cells could have been compensated for by matching an increase in the number of PV-IR basket cells, thus resulting in no net change in PV-IR cells. Other changes in PV-IR cells in the frontal cortex of prenatal cocaine-exposed animals have been noted. An increase in the numbers of primary and secondary dendrites of PV-IR cells in the prefrontal cortex of rabbits exposed to cocaine in utero has been noted previously (Wang et al., 1996; Stanwood et al., 2001). These authors also noted that the number of PV-IR neurons in the frontal cortex did not change in these animals (Wang et al., 1996; Stanwood et al., 2001). Potentially, the increase in the dendritic field of chandelier cells could result in an increased excitation of the inhibitory chandelier cells; although, this increased inhibition would be expected to impact on a reduced population of mPFC pyramidal neurons because of the reduction in the number of GAT-1-IR candle axons in prenatal cocaine-exposed rats.

The loss of an axo-axonic structure is unique because it directly impacts only on a single pyramidal neuron and cannot be compensated for by an increase in postsynaptic receptors. If all things remained the same, the elimination of this inhibitory structure could increase the number of successful axon potentials generated and thus increase its overall excitatory output. Other neurons innervated by this pyramidal neuron would also be affected. Second, an increase in the stimulation of the chandelier cell via enhanced input through the enlarged dendritic field (Wang et al., 1996; Stanwood et al., 2001) or other mechanism would then result in the inhibition of a smaller select population of mPFC pyramidal neurons in the prenatal cocaine-exposed animal. Potentially, prenatal exposure to cocaine could contribute to a situation in which one subset of pyramidal neurons (without GAT-1-IR candles) would be selectively activated, and another subset of pyramidal neurons (with GAT-1-ir candles) would be selectively inhibited. Current theories of the cognitive functioning of the frontal cortex are based on the inhibition of the majority of pyramidal neurons and selective activation of a small network of pyramidal neurons that represent the processed sensory or mnemonic information (Constantinidis et al., 2002; Seamans et al., 2002). The select activation and inhibition of distinct subsets of pyramidal neurons in the $\mathrm{mPFC}$ attributable to changes in axo-axonic structures represents a potential mechanism for the disruption of cortical function that could result in the cognitive deficits reported in prenatal cocaine-exposed rats. In support of this, we recently reported an enhanced activation of the immediate early gene Fos, selectively in the frontal cortex of prenatal cocaine-exposed rats (Morrow et al., 2002a). Because the insult (cocaine exposure) occurred during development, it is not clear that this potential mechanism exists or whether other unknown compensatory changes have occurred. It is unlikely that the alteration in GAT-1-IR axo-axonic structures is the only cocaineinduced insult that contributes to the altered excitatory/inhibitory balance of the mPFC. Changes in the anatomy and function of the mesoprefrontal dopaminergic neurons (Elsworth et al., 2001; Morrow et al., 2001), as well as changes in the postsynaptic dopamine $\mathrm{D}_{1}$ receptors (Friedman et al., 1996; Jones et al., 2000), are also likely to contribute to the dysfunction of the frontal cortex resulting from prenatal cocaine exposure.

Changes in each of the neurotransmitter systems, GABA and dopamine, are likely accompanied by a change in the relationship between these transmitter systems. From experimental data gathered over the last few years, we can speculate on how the cognitive frontal cortex altered by prenatal cocaine exposure functions during a mild environmental stress. This stress would activate intrinsic neurons in the frontal cortex of normal animals and, in response, inhibitory factors including local GABA interneurons. Additionally, dopaminergic projections acting directly and indirectly through GABAergic interneurons would complement GABAergic function to diminish the activation of the majority of prefrontal pyramidal neurons (Morrow et al., 1999a, b). The elimination of activation in the majority of pyramidal neurons, along with a complementary activation of a small group of select pyramidal neurons representing the processed sensory or mnemonic information, is thought to be necessary for proper functioning of the prefrontal cortex during a cognitive task (Constantinidis et al., 2002; Seamans et al., 2002). In the prenatal cocaineexposed animal, the same mild environmental stress would activate pyramidal neurons in the frontal cortex; however, the GABAergic/dopaminergic inhibitory response now appears unable to diminish the activation of the majority of pyramidal neurons (Morrow et al., 2002a). A greater amount of dopamine is released in the frontal cortex of a cocaine-exposed rat during mild stress (Elsworth et al., 2001; Morrow et al., 2001), although there are two known caveats that must be considered. First, there are potentially fewer dopamine (DA) neurons projecting to the frontal cortex (Morrow et al., 2001). This may limit the dynamic potential of the dopaminergic input and could contribute to the exaggerated release of dopamine in response to stress. Second, dopaminergic transmission would occur primarily through the dopamine $\mathrm{D}_{2}$ receptor because of an uncoupling of the dopamine $\mathrm{D}_{1}$ receptor from its G-protein (Friedman et al., 1996; Jones et al., 2000). Recently, Seamans et al. (2002) reported that dopamine modulates GABAergic inhibition of prefrontal pyramidal neurons in a biphasic manner and proposed that during the early $\mathrm{D}_{2}$-dominated phase, the activation of multiple networks of pyramidal neurons representing various sensory information is encouraged and then, during the subsequent $\mathrm{D}_{1}$-dominated phase, the weaker activated networks are eliminated, thus focusing the cognitive function of the prefrontal cortex. The loss of the proposed $\mathrm{D}_{1}$-related actions could contribute to an exaggerated response to environmental stimuli (Morrow et al., 2002a) and poor short-term memory (Morrow et al., 2002b) in the prenatal 
cocaine-exposed rat. However, much more work is necessary to test and likely refine this hypothesis.

How cocaine disrupts the development of the nervous system is not known; however, it seems unlikely that it involves direct actions of cocaine on the axo-axonic cells in the frontal cortex in the rat. The neurogenesis of the GABAergic chandelier cells is $\sim$ E17-E20, with the neurons in the shallower layers in which most of the axo-axonic structures are located, being "born" at the later timepoints. After $\sim 2-3 \mathrm{~d}(\sim \mathrm{E} 20-\mathrm{P} 1)$, the chandelier cells migrated into layers II and III of the frontal cortex of the rat. E20 is the last day of administration of cocaine into the gravid dam. This means that during the development and refining of the axoaxonic projections, which occurs over the first 2-3 weeks after birth (Yan et al., 1997), no cocaine would remain in the pup. Therefore, it seems very unlikely that cocaine had a direct effect on the axon formation of the chandelier cells. We conclude that there are two possible scenarios. First, cocaine acting indirectly through pulsatile increases in extracellular catecholamines or directly on the newborn chandelier cells through unknown means may induce long-term changes in the developing chandelier cells that subsequently result in fewer successful axons per cell. However, there is little or no evidence to support this possibility. Second, cocaine may induce long-term changes in nonchandelier cells that subsequently alter the development of the chandelier cells, resulting in fewer axo-axonic structures. On the basis of the known actions of in utero cocaine, we believe that this second option is more probable.

One potential candidate to undergo long-term alteration by cocaine exposure is the mesoprefrontal DA neurons. Dopamine has demonstrated to have a role as a neurodevelopmental factor. The primary pharmacological action of cocaine is its ability to block the uptake of serotonin, norepinephrine, and dopamine. Experiments indicate that this action of cocaine is present in the developing fetal brain. Cocaine that is given to the pregnant dam can cross the placental barrier (DeVane et al., 1989; Spear et al., 1989a; Wiggins, 1992) and, presumably, can increase dopamine in the fetus in the womb. Disruption of PV-IR interneurons has previously been linked to dopamine in the prenatal cocaine model (Stanwood et al., 2001). Recently, several laboratories, including ours, have documented a number of long-term changes to the midbrain dopamine system as a result of prenatal exposure to cocaine. We have noted that prenatal cocaine exposure diminished the number of $\mathrm{A}_{10}$ dopamine neurons in the adolescent offspring (Morrow et al., 2001). Additionally, the function of the mesoprefrontal dopamine neurons was altered. Using different methods, we have noted that these dopamine neurons are hyperresponsive to environmental stimuli (Elsworth et al., 2001; Morrow et al., 2001). In support of this observation, Minabe and colleagues (Minabe et al., 1992) noted that animals prenatally exposed to cocaine had an increase in the number of spontaneously firing midbrain dopamine neurons. Postsynaptic changes in the dopamine $\mathrm{D}_{1}$ receptor have also been noted. An uncoupling of G-protein from the $\mathrm{D}_{1}$ receptor complex has been observed previously (Friedman et al., 1996; Jones et al., 2000). Together, these data indicate that prenatal exposure to cocaine can result in long-term changes in dopaminergic neurotransmission. Other potential candidates include the noradrenergic and serotonergic systems; however, less evidence exists to support these possibilities. The regional loss of candles was most evident in the ventral aspects of the medial prefrontal cortex at $\sim 3 \mathrm{~mm}$ anterior to bregma. This region receives dopaminergic innervation along with innervation from other cell groups. The dopaminergic innervation of this region has been demonstrated to be more reac- tive to mild stress than dopaminergic innervation to adjacent regions of the medial prefrontal cortex and may represent a distinct subregion within the medial prefrontal cortex of adult animals (Deutch, 1993; Morrow et al., 2002c). Additionally, in adult rats, lower levels of dopamine transporter have been demonstrated in the ventral medial prefrontal cortex compared with the dorsal (Ciliax et al., 1995; Sesack et al., 1998). Although a mechanism is not clear at this time, the dopaminergic neurons projecting to the ventral medial prefrontal cortex may potentially have a unique sensitivity to cocaine in utero and alone, or through interactions with other neurotransmitters, may selectively alter interneuronal development in that region. We hypothesize that the known alterations in the midbrain dopamine systems contribute to the eventual reduction in the number of axo-axonic structures and, subsequently, to the cognitive deficits.

In addition to a reduction in the number of axo-axonic candles, prenatal cocaine exposure is associated with a change in their pattern of distribution. In the saline control rats, the depth of the first candle was reduced in parallel with the reduction in the cortical thickness that occurs from dorsal to ventral in the medial prefrontal cortex. The depth of the first candles in the prenatal cocaine-exposed rats did not follow this pattern but remained at the same depth throughout the cortex. This could indicate that either the pyramidal neurons targeted by the axo-axonic projections failed to fully migrate to the shallower layers, or that the axo-axonic projections are impinging on a different population of pyramidal neurons in the prenatal cocaine-exposed rat. The potential consequences of this subtle rearrangement of the remaining candles are not clear at this time; however, this could potentially contribute to the disruption in cognitive function as noted previously.

In summary, we have documented a reduction in the number, and a change in the organization, of the axo-axonic structures in prenatal cocaine-exposed rats. The changes in these inhibitory structures could disrupt normal neurotransmission in the prefrontal cortex and, in combination with other changes noted with prenatal cocaine exposure, may result in impaired cognition. We hypothesize that these changes in the axo-axonic candles are secondary to changes in the number and function of the mesocortical dopamine neurons that more directly result from the in utero cocaine exposure.

\section{References}

Ciliax BJ, Heilman C, Demchyshyn LL, Pristup ZB, Ince E, Hersch SM, Niznik HB, Levey AI (1995) The dopamine transporter: immunochemical characterization and localization in the brain. J Neurosci 15:1714-1723.

Constantinidis C, Williams GV, Goldman-Rakic PS (2002) A role for inhibition in shaping the temporal flow of information in prefrontal cortex. Nat Neurosci 5:175-180.

DeFelipe J, Hendry SH, Jones EG, Schmechel D (1985) Variability in the terminations of GABAergic chandelier cell axons on initial segments of pyramidal cell axons in the monkey sensory-motor cortex. J Comp Neurol 231:364-384.

Delaney-Black V, Covington C, Templin T, Ager J, Martier S, Sokol R (1998) Prenatal cocaine exposure and child behavior. Pediatrics 102:945-950.

DeVane CL, Simpkins JW, Miller RL, Braun SB (1989) Tissue distribution of cocaine in the pregnant rat. Life Sci 45:1271-1276.

Dow-Edwards D, Mayes L, Spear L, Hurd Y (1999) Cocaine and development: clinical, behavioral, and neurobiological perspectives-a symposium report. Neurotoxicol Teratol 21:481-490.

Deutch AY (1993) Prefrontal cortical dopamine systems and the elaboration of functional corticostriatal circuits: implications for schizophrenia and Parkinson's disease. J Neural Transm Gen Sect 91:197-221.

Elsworth JD, Morrow BA, Roth RH (2001) Prenatal cocaine exposure increases mesoprefrontal dopamine neuron responsivity to mild stress. Synapse 42:80-83. 
Friedman E, Yadin E, Wang HY (1996) Effect of prenatal cocaine on dopamine receptor- $G$ protein coupling in mesocortical regions of the rabbit brain. Neuroscience 70:739-747.

Gabbott PL, Bacon SJ (1996) Local circuit neurons in the medial prefrontal cortex (areas 24a,b,c, 25 and 32) in the monkey, II: quantitative areal and laminar distributions. J Comp Neurol 364:609-636.

Gabbott PL, Dickie BG, Vaid RR, Headlam AJ, Bacon SJ (1997) Localcircuit neurones in the medial prefrontal cortex (areas 25,32 and 24b) in the rat: morphology and quantitative distribution. J Comp Neurol 377:465-499.

Garavan H, Morgan RE, Mactutus CF, Levitsky DA, Booze RM, Strupp BJ (2000) Prenatal cocaine exposure impairs selective attention: evidence from serial reversal and extradimensional shift tasks. Behav Neurosci 114:725-738.

Heffelfinger A, Craft S, Shyken J (1997) Visual attention in children with prenatal cocaine exposure. J Int Neuropsychol Soc 3:237-245.

Heyser CJ, Spear NE, Spear LP (1995) Effects of prenatal exposure to cocaine on Morris water maze performance in adult rats. Behav Neurosci 109:734-743.

Hof PR, Glezer II, Conde F, Flagg RA, Rubin MB, Nimchinsky EA, Vogt Weisenhorn DM (1999) Cellular distribution of the calcium-binding proteins parvalbumin, calbindin, and calretinin in the neocortex of mammals: phylogenetic and developmental patterns. J Chem Neuroanat 16:77-116.

Howard CV, Reed MG (1998) Unbiased stereology: three-dimensional measurement in microscopy, pp 125-130. New York: Springer.

Jones LB, Stanwood GD, Reinoso BS, Washington RA, Wang HY, Friedman $\mathrm{E}$, Levitt $\mathrm{P}$ (2000) In utero cocaine-induced dysfunction of dopamine $\mathrm{D}_{1}$ receptor signaling and abnormal differentiation of cerebral cortical neurons. J Neurosci 20:4606-4614.

Leech SL, Richardson GA, Goldschmidt L, Day NL (1999) Prenatal substance exposure: effects on attention and impulsivity of 6-year-olds. Neurotoxicol Teratol 21:109-118.

Lewis DA (2000) GABAergic local circuit neurons and prefrontal cortical dysfunction in schizophrenia. Brain Res Brain Res Rev 31:270-276.

Mayes LC, Grillon C, Granger R, Schottenfeld R (1998) Regulation of arousal and attention in preschool children exposed to cocaine prenatally. Ann NY Acad Sci 846:126-143.

Minabe Y, Ashby Jr CR, Heyser C, Spear LP, Wang RY (1992) The effects of prenatal cocaine exposure on spontaneously active midbrain dopamine neurons in adult male offspring: an electrophysiological study. Brain Res 586:152-156.

Morrow BA, Elsworth JD, Rasmusson AM, Roth RH (1999a) The role of mesoprefrontal dopamine neurons in the acquisition and expression of conditioned fear in the rat. Neuroscience 92:553-564.

Morrow BA, Elsworth JD, Zito C, Roth RH (1999b) Biochemical and behavioral anxiolytic-like effects of R(+)HA-966 at the level of the ventral tegmental area in rats. Psychopharmacology 143:227-234.

Morrow BA, Elsworth JD, Roth RH (2001) Prenatal exposure to cocaine reduces the number and enhances reactivity of A10 dopaminergic neurons to environmental stress. Synapse 41:337-344.

Morrow BA, Elsworth JD, Roth RH (2002a) Male rats exposed to cocaine in utero demonstrate elevated expression of Fos in the prefrontal cortex in response to environment. Neuropsychopharmacology 26:275-285.

Morrow BA, Elsworth JD, Roth RH (2002b) Prenatal cocaine exposure disrupts non-spatial, short-term memory in adolescent and adult male rats. Behav Brain Res 129:217-223.
Morrow BA, Elsworth JD, Roth RH (2002c) Fear-like biochemical and behavioral responses in rats to the predator odor, TMT, are dependent on the exposure environment. Synapse 46:11-18.

Ong WY, Yeo TT, Balcar VJ, Garey LJ (1998) A light and electron microscopic study of GAT-1-positive cells in the cerebral cortex of man and monkey. J Neurocytol 27:719-730.

Peters A, Harriman KM (1990) Different kinds of axon terminals forming symmetric synapses with the cell bodies and initial axon segments of layer II/III pyramidal cells, I: morphometric analysis. J Neurocytol 19:154-174.

Peters A, Harriman KM (1992) Different kinds of axon terminals forming symmetric synapses with the cell bodies and initial axon segments of layer II/III pyramidal cells, III: origins and frequency of occurrence of the terminals. J Neurocytol 21:679-692.

Peters A, Proskauer CC, Ribak CE (1982) Chandelier cells in rat visual cortex. J Comp Neurol 206:397-416.

Peters A, Sethares C, Harriman KM (1990) Different kinds of axon terminals forming symmetric synapses with the cell bodies and initial axon segments of layer II/III pyramidal cells, II: synaptic junctions. J Neurocytol 19:584-600.

Pierri JN, Chaudry AS, Woo TW, Lewis DA (1999) Alterations in chandelier neuron axon terminals in the prefrontal cortex of schizophrenic subjects. Am J Psychiatry 156:1709-1719.

Seamans JK, Gorelova N, Durstewitz D, Yang CR (2002) Bidirectional dopamine modulation of GABAergic inhibition in prefrontal cortical pyramidal neurons. J Neurosci 21:3628-3638.

Sesack SR, Hawrylak VA, Matus C, Guido MA, Levey AI (1998) Dopamine axon varicosities in the prelimbic division of the rat prefrontal cortex exhibit sparse immunoreactivity for the dopamine transporter. J Neurosci 18:2697-2708.

Spear LP, Frambes NA, Kirstein CL (1989a) Fetal and maternal brain and plasma levels of cocaine and benzoylecgonine following chronic subcutaneous administration of cocaine during gestation in rats. Psychopharmacology 97:427-432.

Stanwood GD, Washington RA, Shumsky JS, Levitt P (2001) Prenatal cocaine exposure produces consistent developmental alterations in dopamine-rich regions of the cerebral cortex. Neuroscience 106:5-14.

Volk D, Austin M, Pierri J, Sampson A, Lewis D (2001) GABA transporter-1 mRNA in the prefrontal cortex in schizophrenia: decreased expression in a subset of neurons. Am J Psychiatry 158:256-265.

Wang XH, Levitt P, Grayson DR, Murphy EH (1995) Intrauterine cocaine exposure of rabbits: persistent elevation of GABA-immunoreactive neurons in anterior cingulate cortex but not visual cortex. Brain Res 689:32-46.

Wang XH, Jenkins AO, Choi L, Murphy EH (1996) Altered neuronal distribution of parvalbumin in anterior cingulate cortex of rabbits exposed in utero to cocaine. Exp Brain Res 112:359-371.

Wiggins RC (1992) Pharmokinetics of cocaine in pregnancy and effects on fetal maturation. Clin Pharmcokinet 22:85-93.

Woo TU, Whitehead RE, Melchitzky DS, Lewis DA (1998) A subclass of prefrontal gamma-aminobutyric acid axon terminals are selectively altered in schizophrenia. Proc Natl Acad Sci USA 95:5341-5346.

Yan XX, Cariaga WA, Ribak CE (1997) Immunoreactivity for GABA plasma membrane transporter, GAT-1, in the developing rat cerebral cortex: transient presence in the somata of neocortical and hippocampal neurons. Brain Res Dev Brain Res 99:1-19. 